sen, 1974). Behringwerke use venom of $E$. carinatus pyramidum (Geoffroy Saint Hilaire) from the Sudan, the electrophoretic pattern of which is very similar to that of Ethiopian venom (Behringwerke, 1974). Thus, geographical differences in venoms are unlikely to explain the difference in potency of these two antivenoms. A higher titre of specific antibody can be expected in monospecific as compared with polyspecific antivenom, but this difference should be overcome by using a higher dose. Our findings suggest that S.A.I.M.R. antivenom contains a specifically higher titre of neutralizing antibody directed against the procoagulant and perhaps the necrosis-inducing venom fractions.

This work was supported by the Wellcome Trust and H.A.R. was the recipient of a Welloome Research Travel Grant. Cotton and Agricultural Processors Ltd. (formerly B.C.G.A., Nigeria, Ltd.) at Zaria and Gombe provided invaluable help with housing and communications.

Dr. P. A. Christensen, Director of the South African Institute for Medical Research, generously donated supplies of S.A.I.M.R. antivenom for this study.

We thank the Chief Medical Officer, North Eastern State,
Nigeria (Dr. S. Hussain) for permission to conduct the studies at Gombe and Kaltungo General Hospitals, the medical superintendents of these hospitals (Drs. S. H. A. Lofty and I. F. Shoukry), the chief dispenser, Billiri-Kufai E.C.W.A. Dispensary (the late Mallam Sabo Tukub), and their staffs for help.

\section{References}

Ahuja, M. L., and Singh, G. (1951). Indian fournal of Medical Research, 42, 661

Arnett, C. (1971). Personal communication.

Behringwerke (1974). Personal communication.

Biggs, R. (1972). Human Blood Coagulation, Haemostasis and Thrombosis. Oxford, Blackwell.

Christensen, P. A. (1974). Personal communication.

Greenwood, B. M., et al. (1974). In preparation.

Kornalik, F., and Taborska, E. (1973). In Animal and Plant Toxins, ed. E. Kaiser, 99 . Munich, Goldmann.

Latifi, M. (1973). In Proceedings of the Ninth International Congress on Tropical Medicine and Malaria, abstract 107. Athens, 14-21 October.

Reid, H. A., Thean, P. C., and Martin, W. J. (1963 a). British Medical fournal, 2, 1378 .

Reid, H. A., Chan, K. E., and Thean, P. C. (1963 b). Lancet, 1, 621.

Salou, G. (1951). Medecine Tropicale, 11, 655.

Thomson, J. M. (1970). A Practical Guide to Blood Coagulation and Haemostasis. London, Churchill.

Warrell, D. A., et al. (1974). In preparation.

\title{
Indications for Systemic Antibiotic Prophylaxis in Neutropenic Patients
}

\author{
A. S. D. SPIERS, M. H. N. TATTERSALL, H. GAYA
}

British Medical fournal, 1974, 4, 440-441

\section{Summary}

Four patients with malignant blood disease and neutropenia developed apparently localized infective lesions of the skin or conjunctiva from which Pseudomonas aeruginosa was cultured. In spite of appropriate local antimicrobial treatment all patients developed pseudomonas septicaemia within a short period and three died. It is argued that the isolation of pseudomonads from apparently localized lesions in patients with compromised antibacterial defences is an indication for systemic antibiotic therapy.

\section{Introduction}

Few subjects are more contentious than the prophylactic use of antibiotics. Many studies have shown that the widespread use of prophylactic antibiotics may lead to more rather than less infection. In the immunodepressed or neutropenic patient chemoprophylaxis may delay the acquisition of infection, but when infection does occur in patients receiving antibiotics in this way it usually is caused by antibiotic-resistant organisms. Evidence favouring the use of prophylactic systemic antibiotics has been reported in patients subjected to caesarean section (Gibbs et al., 1972)

M.R.C. Leukaemia Unit and Department of Bacteriology, Roya Postgraduate Medical School, London W12 0HS

A. S. D SPIERS, PH.D. F.R.A.C.P., Consultant Physician

M. H. N. TATTERSALL, M.D., M.R.C.P., M.R.C. Clinical Research Fellow

H. GAYA, M.B., M.R.C.PATH., Consultant Bacteriologist (Present appointment: Reader in Bacteriology, Wright-Fleming Institute, London W2 1PG) and after removal of a perforated appendix (Magarey et al., 1971). In both groups of patients prophylactic antibiotics substantially reduced the infection rate, but the diagnosis of infection in both studies was based mainly on clinical criteria with bacteriological confirmation in only a few patients. After open heart sungery it is customary to administer prophylactic systemic antibiotics but the value of this policy has not been clearly established.

In patients with malignant blood disease and neutropenia infection is the leading cause of death (Hersh et al., 1965; Levine et al., 1972). The natural history of infection in these patients is one of rapid deterioration, and death often occurs before the infecting organism has been isolated (Schimpff et al., 1971). This has prompted us to advocate the empirical use of antibiotic combinations for episodes of presumed infection in these patients (Tattersall et al., 1972; Gaya et al., 1973; Tattersall et al., 1973), and this has been the subject of a recent symposium (European Fournal of Cancer, 1973). At present an international trial is in progress to establish which of three combinations of two antibiotics is the most effective initial therapy for presumed infection in this type of patient. Like other groups we are studying the prophylactic effect of decontaminating the intestinal contents by the oral administration of nonabsorbable antibiotics with the aim of reducing the incidence of septicaemias of enteric onigin. The use of prophylactic systemic antibiotics in patients with malignant blood disease and neutropenia is a separate consideration, and until now this has not been our policy. From routine surveillance cultures in certain patients, however, we have observed the presence of coliform organisms on the skin. These were not eradicated by local antiseptic treatment and were eventually the cause of septicaemia.

We describe here four patients with malignant blood disease whose clinical course prompts us to recommend the use of prophylactic systemic antibiotics in certain clearly 
defined circumstances. In each case Pseudomonas aeuruginosa was cultured from an apparently localized site at a time when the patient was afebrile and well. In spite of appropriate local antimicrobial treatment each patient over the course of a few days became febrile and unwell. Ps. aeruginosa was isolated from their blood cultures. In spite of systemic antibiotics which were begun when symptoms appeared three of the four patients died of the infection.

\section{Case Reports}

Case 1.-A 50-year-old man with acute myeloblastic leukaemia diagnosed four months previously was admitted to hospital in relapse. He was not receiving corticosteroid therapy. He was afebrile and had no symptoms of systemic infection but had a painful left axilla containing an infected hair follicle with erythema and induration. His peripheral blood neutrophil count was $150 / \mathrm{mm}^{3}$ and a swab from the axillary skin grew $P$ s. aeruginosa. The lesion was treated successively with topical aqueous chlorhexidine gluconate $(0.1 \% \mathrm{w} / \mathrm{v}), 4 \% \mathrm{w} / \mathrm{v}$ chlorhexidine gluconate in detergent base (Hibiscrub), $0.5 \% \mathrm{w} / \mathrm{v}$ chlorhexidine gluconate in $70 \% \mathrm{v} / \mathrm{v}$ industrial methylated spirit, and $1 \% \mathrm{w} / \mathrm{w}$ chlorhexidine cream, twice daily under occlusive dressing:. Five days after admission he was pyrexial and unwell. Blood cultures were taken and system:c carbenicillin ( $7 \cdot 5 \mathrm{~g}$ :ix hourly), gentamicin (40 mg six hourly), cephalothin, and lincomycin were begun empirically. Subsequently two blocd cultures grew pseudemonads. A left axillary absces- was incised on the third day of antibiotic therapy and pseudomonads were cultured from the pus. He became afebrile on the eighth day of the antibiotic therapy. Antibiotics were stcpped on the 14th day and he remained well. Swabs of the axillary skin on the 10th day of topical chlorhexidine treatment (which wa- the fifth day of systemic antibiotic treatment) showed no bacterial growth.

Case 2.-A 35-year-old woman was diagnosed as having histiocytic medullary reticulosis one year previously. She had been treated for several months with prednisone by mouth and had been admitted to hospital with hyperglycaemia and glycosuria. She was afebrile, but an infected hair follicle was found in a sore right axilla. The peripheral blood neutrophil count was $500 / \mathrm{mm}^{3}$. On a presumptive diagnosis of staphylococcal infection initial therapy was begun with co-trimoxazole, reduction in the dose of prednisone, and twice-daily dressings of $3 \%$ hexachlorphane. A swab of the axillary $\mathrm{kin}$ was taken and subsequently grew pseudomonads. Two days after admission she was well but pyrexial $\left(38.3^{\circ} \mathrm{C}\right)$ and the axillary lesion was larger though less tender. One week later she was afebrile but had a discharging boil from which pseudomonads were cultured. The neutrophil count was now $150 / \mathrm{mm}^{3}$. Eleven day- after admission she was febrile and unwell and blood cultures grew Ps. aeruginosa. Because she had a severe penicillin allergy she was treated with gentamicin ( $80 \mathrm{mg}$ eight hourly) and polymyxin B (500,000 units six hourly). Though the pseudomonads were sensitive to these antibiotics in vitro she did not respond to treatment and blood cultures remained positive two days after starting trea'ment. She died on the fifth day of antibiotic therapy.

Case 3.-This 52-year-old man with histiocytic lymphoma had a sore left eye diagnosed by the ophthalmologist as keratoconjunctivitic. A conjunctival swab was taken and chloramphenicol eye drops were begun. Subsequently the swab grew Ps. aeruginosa. The peripheral blood neutrophil count was $1.200 / \mathrm{mm}^{3}$. The day after presentation he was afebrile but had a slight cough and Ps. aeruginosa was cultured from his sputum. Before this culture result was available treatment was begun with cloxacillin and gentamicin (80 mg eight hourly). The next day he was ill and febrile and Ps. acruginosa was grown from a blood culture. Despite the antibiotic therapy he deteriorated rapidly and died.

Case 4.-A 10-year-old boy with acute myeloblastic leukaemia of six months' duration was admitted in relapse with a painful left thigh on which was a necrotic ulcer. Swabs from this lesion grew Ps. aeruginosa. The neutrophil count was $3,600 / \mathrm{mm}^{3}$ but with antileukaemic chemotherapy later fell to $40 / \mathrm{mm}^{3}$. Twice-daily dressings of half-strength eusol were applied to the thigh and supplemented by Polybactrin (neomycin-polymyxin B-bacitracin) aerosol. Though in 10 days the ulcer seemed to be clean and was healing and the patient was well pseudomonads were repeatedly grown from swabs of the lesion. A week later he became ill with a temperature of $40^{\circ} \mathrm{C}$. Blood cultures were taken and parenteral penicillin, methicillin, carbenicillin, and kanamycin were begun empirically. The blood cultures grew Ps. aeruginosa and on the fourth day of antibiotic treatment gentamicin ( $25 \mathrm{mg}$ six hourly) was substituted for the kanamycin, while carbenicillin ( $5 \mathrm{~g}$ six hourly) was continued. Temporary slight improvement was followed by deteris"ation and he died on the 14th day of antibiotic treatment.

\section{Discussion}

Many factors are known which contribute to the increased susceptibility of leukaemia patients to septicaemia. In patients with normal bone marrow an infected hair follicle does not constitute an indication for systemic antibiotics. In patients with granulocytopenia the ability of the host to confine invading organisms to a local lesion is reduced, and this may partly explain the relationship which has been established between the risk of infection and numbers of circulating granulocytes (Bodey et al., 1966). In patients with leukaemia not only may granulocytopenia exist but also the function of the few granulocytes present may be severely impaired. Moreover, many of these patients may be receiving corticosteroids systemically, another factor believed to predispose to infection. Thus the ability of patients with malignant blood diseases and neutropenia to confine and subsequently eradicate a localized infection is greatly reduced.

Colonization of the skin with Gram-negative organisms has been noted in severely ill patients (Stratford et al., 1968; Schimpff et al., 1972 a). The reason for this disturbance of the skin flora is not known and our experience is that no form of local treatment eradicates these organisms. In patients with leukaemia whose haematological response to therapy is good Gram-negative skin colonization may be only a transient problem, being corrected as their disease enters remission. As local treatment with antiseptics or topical antibiotics does not eradicate the organisms systemic antibiotic treatment must be considered. On the other hand, though faecal carriage of Ps. aeruginosa in leukaemia patients has been implicated as a cause of rectal abscesses (Schimpff et al., 1972 b), oral non-absorbed-that is, topical-antibiotics often do eradicate the organism from the stools, and subsequent pseudomonas septicaemia is unlikely.

Our cases illustrate that the presence of Ps. aeruginosa in cultures from localized skin lesions is a good indication that pseudomonas septicaemia may supervene in spite of "appropriate" local treatment. We believe that patients with compromised bone marrow and granulocyte function in whom pseudomonads are cultured from the skin should be treated "prophylactically" with the appropriate systemic antibiotics, as indicated by sensitivity tests. Indeed it may be argued that this is not prophylaxis since infection is already present. Ideally, daily granulocyte transfusions may be the most appropriate treatment for localized lesions in granulocytopenic patients, but at present this form of treatment is available at only a few centres and even then is dictated by the availability of suitable granulocyte donors.

\section{References}

Bodey, G. P., et al. (1966). Annals of Internal Medicine, 64, 328. European fournal of Cancer, 1973, 9, No. 6.

Gaya, H., et al. (1973). European fournal of Cancer, 9. 401.

Gibbs, R. S., DeCherney, A. H., and Schwartz, R. H. (1972). American Fournal of Obstetrics and Gynecology, 114, 1048.

Hersh, E. M., et al. (1965). Fournal of the American Medical Association, 193, 105.

Levine, A. S., Graw, R. G., and Young, R. C. (1972). Seminars in Hematology, 9, 141 .

Magarey, C. J., et al. (1971). Lancet, 2, 179.

Schimpff, S., et al. (1971). New England fournal of Medicine, 284, 1061.

Schimpff, S. C., et al. (1972 a). Annals of Internal Medicine, 77, 707.

Schimpff, S. C., Wiernik, P. H., and Block, J. B. (1972 b). Lancet, 2, 844.

Stratford, B., et al. (1968). Lancet, 1, 68.

Tattersall, M. H. N., Spiers, A. S. D., and Darrell, J. H. (1972). Lancet, 1,

162. 